\title{
Cross-cultural adaptation and validation of the Portuguese version of the Knee Outcome Survey-Activities of Daily Living Scale (KOS-ADLS)
}

\author{
Rui Soles Gonçalves • Jan Cabri • João Páscoa Pinheiro
}

Received: 9 May 2008 / Revised: 28 July 2008 / Accepted: 6 August 2008 / Published online: 5 September 2008

(C) Clinical Rheumatology 2008

\begin{abstract}
The objective of this study was to cross-culturally adapt and validate the Portuguese version of the Knee Outcome Survey-Activities of Daily Living Scale (KOSADLS). This version was obtained with forward/backward translations, consensus panels and pre-testing. The Portuguese KOS-ADLS and Medical Outcomes Study, 36-item Short Form (SF-36) questionnaires, visual analogue scales (VAS) of pain, disability and discomfort, and a form for patient's characteristics were administered to 168 subjects with knee osteoarthritis (OA). Reliability was acceptable (Cronbach's alpha $=0.91 ; \mathrm{ICC}=0.97$ ). There were significant correlations with SF-36 physical component subscales, all VAS, and duration of knee OA. The subjects with bilateral knee OA and that need walking aids obtained lower scores $(p<0.001)$. No floor/ceiling effects were detected. Responsiveness to physical therapy was showed (standardized effect size $=0.62$; standardized response mean $=1.02$ ). The Portuguese KOS-ADLS evidenced acceptable reliability, validity, floor/ceiling effects, and responsiveness.
\end{abstract}

R. Soles Gonçalves · J. Cabri

Faculty of Human Kinetics, Center for Research in Physiotherapy,

Technical University of Lisbon,

Lisbon, Portugal

R. Soles Gonçalves $(\square)$

Superior School of Health Technology of Coimbra,

Polytechnic Institute of Coimbra,

Rua 5 de Outubro, S. Martinho do Bispo, Apartado 7006,

3040-162 Coimbra, Portugal

e-mail: ruigoncalves@estescoimbra.pt

J. Páscoa Pinheiro

Faculty of Medicine, University of Coimbra,

Coimbra, Portugal
Keywords Health status $\cdot$ Knee $\cdot$ KOS-ADLS . Osteoarthritis

\section{Introduction}

Patient-oriented questionnaires on health-related quality of life, health status, and functional status are widely used to evaluate health care outcomes. These tools provide significant information about the perceived impact of specific diseases and conditions on individuals [1, 2]. In the context of knee OA generic (e.g., SF-36 [3]; EuroQol [4]), diseasespecific (e.g., Western Ontario and McMaster Universities Osteoarthritis Index [5]; Lequesne Algofunctional Index [6]) and site-specific instruments (e.g., Knee injury and Osteoarthritis Outcome Score [7]; KOS-ADLS [8]) are most common. The greater number of existing patient selfadministered questionnaires have their origins in AngloAmerican scientific literature [9]. The use of these instruments in other cultures or different languages requires a crosscultural adaptation and validation [10].

The KOS-ADLS was developed for the evaluation of patients with knee disorders, including knee OA, assessing the symptoms (pain, crepitus, stiffness, swelling, instability, and weakness) and the functional disability that could be felt during the performance of daily living activities (walking, stairs ascending/descending, standing, kneeling, squatting, and chair sitting/rising) [8]. It has the advantage of being easily used to follow patients with different knee problems throughout the life span.

The objective of this paper was to realize the translation and cultural adaptation of the KOS-ADLS to the Portuguese language (for use in Portugal) and to study its reliability, validity, floor/ceiling effects, and responsiveness in patients with knee OA. 


\section{Materials and methods}

Cross-cultural adaptation

This process followed previously established guidelines [9, 11]. The American-English KOS-ADLS was used as starting point with permission from the author [8]. This version was translated into Portuguese separately by two translators. The two resultant translations were used in a first consensus panel to obtain the first preliminary version. This consensus version was translated back to AmericanEnglish language separately by another two translators without previous contact with the original version. The translations and back translations were used in a second consensus panel to obtain the second preliminary version. In order to assure that all items of the questionnaire were comprehensible, this consensus version was filled out by 10 subjects with specific knee conditions. A third consensus panel took place to obtain the final version of Portuguese KOS-ADLS questionnaire.

\section{Validation study}

\section{Subjects}

The sample consisted of patients with symptomatic knee OA fulfilling the clinical and radiographic criteria of the American College of Rheumatology [12], attending 10 physical therapy outpatient clinics in Portugal during a 6-month period. Subjects were selected after obtaining informed consent and checking the inclusion and exclusion criteria. To be included in the study, subjects had to require physical therapy treatments (related to knee OA) with an expected duration of at least 4 weeks and experience knee pain from OA with a score of at least $30 \mathrm{~mm}$ on a 0 to $100 \mathrm{~mm}$ VAS. Subjects were excluded if they had attended physical therapy treatments (related to knee OA) in the previous 30 days, had other lower limb osteoarthropathy, neurological disease, or any other disabling condition or if they were unable to read and write Portuguese fluently. All physical therapy outpatient clinics obtained approval from their respective review boards.

\section{Measurements}

Measurements were performed at the clinics. The whole sample was assessed in the first clinic visit and again $48 \mathrm{~h}$ later. In six of the 10 physical therapy outpatient clinics all subjects were assessed once more after 4 weeks of physical therapy treatments. Data were collected in a questionnaire format using the translated patient self-completion measures.

The KOS-ADLS [8] contains 17 items which assess the level of knee function through a final global score that ranges from 0 (lower level of function) to 100 (higher level of function). The American-English KOS-ADLS satisfies standard criteria for reliability, validity, and responsiveness $[8,13]$.

The SF-36 [3] contains 36 items that cover eight subscales: physical functioning, role-physical, bodily pain, general health, vitality, social functioning, role-emotional, and mental health. A score, from 0 (worst possible health status) to 100 (best possible health status), is separately produced for each subscale. The SF-36 was cross-culturally adapted to the Portuguese language [14], and this version was validated [15].

Three VAS were used to assess the intensity of knee pain, degree of disability related with the knee, and the degree of discomfort in walking, respectively. All the VAS ranged from 0 (no problems) to $100 \mathrm{~mm}$ (extreme problems). The VAS has been found to be reliable and valid in evaluating subjects with knee-specific conditions [16].

A form was used to acquire subject information on gender, age, body mass index, duration of knee OA (from diagnosis of knee OA), involved knee (knee with OA), and walking aids.

\section{Statistical analyses}

Reliability Internal consistency was assessed using Cronbach's alpha and corrected item-total scale correlations. An alpha value between 0.70 and 0.95 was considered as acceptable reliability [17]. Corrected item-total scale correlation of 0.30 or higher was considered acceptable for each item in the scale [18]. Reproducibility over $48 \mathrm{~h}$ was evaluated using ICC. A reliability coefficient of 0.70 or higher was considered as acceptable for group comparisons [10].

Validity Construct validity was tested by relating KOSADLS scores with variables that could be expected to have an association with them (SF-36 physical component subscales, all VAS, and duration of knee OA) and by comparing KOS-ADLS scores obtained by different subgroups based on grouping variables that could be expected to produce statistically significant differences (involved knee and walking aids). Construct validity was tested using Spearman's correlation and Mann-Whitney test. Spearman's correlation coefficients were interpreted as follows: excellent relationship, $\geq 0.91$; good, 0.90-0.71; fair, 0.700.51 ; weak, 0.50-0.31, little or none, $\leq 0.30$ [19]. A $p$ value of 0.05 was taken as the level of significance.

Floor/ceiling effects Floor/ceiling effects were considered present if more than $15 \%$ of the participants receive either the lowest-possible or highest-possible score of the scale [17].

Responsiveness Responsiveness to 4 weeks of physical therapy was assessed using the standardized effect size and 
standardized response mean. The effect sizes were calculated as described by Husted et al. [20]. A value of 0.80 or higher was considered high responsiveness [20].

All statistical analyses were conducted with Statistical Package for the Social Sciences, SPSS ${ }^{\circledR} 15.0$ for Windows ${ }^{\circledR}$ (SPSS Inc., Chicago, IL, USA).

\section{Results}

Cross-cultural adaptation

The second preliminary version of Portuguese KOS-ADLS questionnaire was well-accepted in the pre-test. All the questions and response options were considered satisfactorily comprehensible by the subjects. Therefore, this version was not subjected to any additional modification and was used in the validation study.

Validation study

\section{Subjects}

A total of 168 patients (Table 1) were included in the reliability, validity, and floor/ceiling effects assessment, of

Table 1 Characteristics of the subjects $(N=168)$

\begin{tabular}{ll}
\hline Characteristics & Baseline data \\
\hline Gender & \\
Female & $126(75.0)$ \\
Age (years) & $67.8 \pm 7.8$ \\
Body mass index $\left(\mathrm{kg} / \mathrm{m}^{2}\right)$ & $28.5 \pm 3.8$ \\
Duration of knee OA (years) & $9.3 \pm 6.7$ \\
Involved knee (knee with OA) & \\
Bilateral & $108(64.3)$ \\
Walking aids & \\
No aids necessary & $131(78.0)$ \\
KOS-ADLS final global score (points) & $45.0 \pm 17.3$ \\
VAS (mm) & $68.9 \pm 20.1$ \\
Pain & $66.0 \pm 22.7$ \\
Disability & $70.6 \pm 21.0$ \\
Discomfort & \\
SF-36 subscales scores (points) & $37.7 \pm 21.0$ \\
Physical functioning & $33.7 \pm 24.7$ \\
Role-physical & $29.4 \pm 18.8$ \\
Bodily pain & $36.6 \pm 17.3$ \\
General health & $36.3 \pm 24.9$ \\
Vitality & $58.1 \pm 28.5$ \\
Social functioning & $44.9 \pm 29.0$ \\
Role-emotional & $50.1 \pm 27.1$ \\
Mental health & \\
\hline
\end{tabular}

Quantitative variables, mean \pm standard deviation; categorical variables, frequency (percentage) which 107 (64\%) were also included in the responsiveness assessment.

\section{Reliability}

Cronbach's alpha coefficient was 0.91 and corrected itemtotal scale correlations ranged from 0.40 to 0.78 . ICC was 0.97 for the KOS-ADLS final global score and between 0.88 and 1.00 for the 17 items of the questionnaire.

\section{Validity}

There were significant correlations with SF-36 physical component subscales, all VAS, and duration of knee OA (Table 2). The subjects with bilateral knee OA and that need walking aids obtained lower scores $(p<0.001)$.

\section{Ceiling/floor effects}

None of the subjects reached the worst possible or best possible scale scores.

\section{Responsiveness}

Results are summarized in Table 3.

\section{Discussion}

We translated and culturally adapted the KOS-ADLS to the Portuguese language and presented evidence of its reliabil-

Table 2 Relationship between KOS-ADLS final global score and SF36 physical component subscales scores, pain, disability, discomfort and duration of knee OA $(N=168)$

\begin{tabular}{lccc}
\hline & & & $\begin{array}{c}\text { KOS-ADLS } \\
\text { final global score }\end{array}$ \\
\hline SF-36 physical component & Physical & $r$ & 0.69 \\
subscales scores & functioning & $p$ & $<0.001$ \\
& Role-physical & $r$ & 0.60 \\
& Bodily pain & $p$ & $<0.001$ \\
& & $r$ & 0.55 \\
GAS & General heath & $r$ & $<0.001$ \\
& & $p$ & 0.34 \\
& Pain & $r$ & -0.53 \\
& Disability & $p$ & $<0.001$ \\
& & $p$ & -0.55 \\
& Discomfort & $r$ & -0.56 \\
Duration of & & $p$ & $<0.001$ \\
knee OA & & $r$ & -0.23 \\
& & $p$ & 0.002 \\
\hline
\end{tabular}

Spearman's correlation coefficient (KOS-ADLS and SF-36 are $0-100$ points, worst to best; VAS is $0-100 \mathrm{~mm}$, best to worst) 
Table 3 Standardized effect size and standardized response mean $(N=107)$
Standardized effect

size (effect size I)
Standardized response mean (effect size II)

\begin{tabular}{llll}
\hline KOS-ADLS final global score & 0.62 & 1.02 \\
SF-36 subscales scores & Physical functioning & 0.38 & 0.46 \\
& Role-physical & 0.45 & 0.66 \\
& Bodily pain & 1.00 & 1.11 \\
& General heath & 0.31 & 0.48 \\
& Vitality & 0.49 & 0.59 \\
& Social functioning & 0.24 & 0.30 \\
& Role-emotional & 0.31 & 0.45 \\
& Mental health & 0.42 & 0.57
\end{tabular}

ity, validity, floor/ceiling effects, and responsiveness in patients with knee OA.

The cross-cultural adaptation process resulted in a reasonably comprehensible Portuguese version of the KOS-ADLS. Bizzini and Gorelick [21] also reported the absence of particular difficulties during the translation and cultural adaptation of a German KOS-ADLS. The intelligible wording of all questions and response options seems to facilitate the selection of commonly used words in others cultures or languages and, consequently, to make the questionnaire easy to understand by people with knee problems.

High Cronbach's alpha coefficient and acceptable corrected item-total scale correlations demonstrated that the 17 items of the Portuguese KOS-ADLS are adequately correlated with each other. The results for internal consistency were similar to those obtained by the original American-English version in patients with several disorders of the knee (Cronbach's alpha coefficients of 0.92-0.93 and corrected item-total scale correlations ranging from 0.19 to 0.81 ) [8], and by a German version in non-operative and postoperative knee patients (Cronbach's alpha coefficient of 0.89 and corrected item-total scale correlations ranging from 0.83 to 0.88 ) [21]. High ICC for the final global score and for the 17 items of the questionnaire revealed that the stability of the Portuguese KOS-ADLS over time was acceptable. Similar results were obtained in other studies, 0.97 by Irrgang et al. [8], 0.93 by Marx et al. [13] and $0.94-$ 0.97 by Bizzini and Gorelick [21]. The instrument appears to provide internally consistent and reproducible results either for patient groups with knee OA or for less homogeneous patient groups (various knee injuries and disorders) $[8,13,21]$.

The hypotheses for construct validity were confirmed. As anticipated, the Portuguese KOS-ADLS was associated with SF-36 physical component subscales, intensity of pain, degree of disability, degree of discomfort, and duration of knee OA; and was able to discriminate groups of patients based on involved knee and walking aids. The construct validity of other questionnaire versions had already been supported by correlation with other self-reported measures and performance-based tests of function [8, 13, 21]. The scores of the original American-English version exhibited fair to good correlations with Lysholm Knee Scale $(r=$ $0.78-0.86)$ and global rating of function $(r=0.66-0.75)$ [8]. Marx et al. [13] also found good to excellent positive correlations with various knee outcome measures $(r=0.68$ $0.85)$ and SF-36 physical component scale $(r=0.77)$. A German KOS-ADLS showed moderate correlations with selected functional tests (timed get up and go and timed stairs ascending/descending), and high correlations with VAS of pain intensity [21].

A lowest score of 15.0 points and a highest score of 91.3 points were obtained for the Portuguese KOS-ADLS, which reveals the absence of floor/ceiling effects. Marx et al. [13] also found no floor/ceiling effects for athletic patients. The questionnaire covers a wide range of symptoms and daily living activities which can contribute to minimize the floor/ ceiling effects. In the study of Irrgang et al. [8] minimum ceiling effect (0.9-5.6\%) was detected but only after 1, 4, and 8 weeks of physical therapy. Bizzini and Gorelick [21] also found minimum ceiling effect (one individual). This may be attributed to the inclusion of patients with less severe knee conditions.

The results of the responsiveness assessment showed that the Portuguese KOS-ADLS was able to detect changes over time. Medium standardized effect size and large standardized response mean were found after 4 weeks of physical therapy treatments. With the exception of bodily pain, all SF-36 subscales presented lower standardized effect size and standardized response mean. This confirms that specific measures tend to be more responsive than generic measures [22, 23]. Irrgang et al. [8] found a standardized effect size of $0.44,0.94$, and 1.26 after 1,4 , and 8 weeks of physical therapy treatments, respectively, in patients with knee injury and OA [8]. Marx et al. [13] also reported a large standardized response mean (1.1) after a minimum of 3 months of operative or non-operative treatments in patients with primary disorders of the knee.

Some limitations of this study should be highlighted. In fact, only patients with knee OA attending physical therapy 
treatments in outpatient clinics were recruited. This sample does not represent the whole population of Portuguese patients with knee OA. Besides, KOS-ADLS is a sitespecific instrument that could be used to assess not only patients with knee OA but also patients with other kneespecific clinical conditions. Because the reliability, validity, and responsiveness of patient-oriented outcome measures are population-specific [2], the psychometric properties evidenced by the Portuguese KOS-ADLS in this study may be somewhat different in other populations with knee injuries.

In conclusion, the Portuguese version of the KOS-ADLS obtained in this study evidenced reliability, validity, floor/ ceiling effects, and responsiveness comparable to the original American-English version. Further testing is required in other populations (e.g., patients with patellofemoral pain, ligamentous, and meniscal injuries).

Acknowledgements The authors would like to thank Dr. Nancy Hamilton (University of Northern Iowa, USA) for the English editing of the manuscript.

Disclosures None.

\section{References}

1. Beaton DE, Schemitsch E (2003) Measures of health-related quality of life and physical function. Clin Orthop Relat Res 413:90-105

2. Fitzpatrick R, Davey C, Buxton MJ et al (1998) Evaluating patient-based outcome measures for use in clinical trials. Health Technol Assess 2:i-iv, 1-74

3. Ware JE Jr, Sherbourne CD (1992) The MOS 36-item short-form health survey (SF-36). I. Conceptual framework and item selection. Med Care 30:473-483

4. (1990) EuroQol-a new facility for the measurement of health-related quality of life. The EuroQol Group. Health Policy 16:199-208

5. Bellamy N, Buchanan WW, Goldsmith CH et al (1988) Validation study of WOMAC: a health status instrument for measuring clinically important patient relevant outcomes to antirheumatic drug therapy in patients with osteoarthritis of the hip or knee. $\mathrm{J}$ Rheumatol 15:1833-1840

6. Lequesne MG, Mery C, Samson M et al (1987) Indexes of severity for osteoarthritis of the hip and knee. Validation-value in comparison with other assessment tests. Scand J Rheumatol Suppl 65:85-89

7. Roos EM, Roos HP, Lohmander LS et al (1998) Knee injury and osteoarthritis outcome score (KOOS) - development of a selfadministered outcome measure. J Orthop Sports Phys Ther 28:88-96

8. Irrgang JJ, Snyder-Mackler L, Wainner RS et al (1998) Development of a patient-reported measure of function of the knee. J Bone Joint Surg Am 80:1132-1145

9. Guillemin F, Bombardier C, Beaton D (1993) Cross-cultural adaptation of health-related quality of life measures: literature review and proposed guidelines. J Clin Epidemiol 46:1417-1432

10. (2002) Assessing health status and quality-of-life instruments: attributes and review criteria. Qual Life Res 11:193-205

11. Beaton DE, Bombardier C, Guillemin F et al (2000) Guidelines for the process of cross-cultural adaptation of self-report measures. Spine 25:3186-3191

12. Altman R, Asch E, Bloch D et al (1986) Development of criteria for the classification and reporting of osteoarthritis. Classification of osteoarthritis of the knee. Diagnostic and Therapeutic Criteria Committee of the American Rheumatism Association. Arthritis Rheum 29:1039-1049

13. Marx RG, Jones EC, Allen AA et al (2001) Reliability, validity, and responsiveness of four knee outcome scales for athletic patients. J Bone Joint Surg Am 83-A:1459-1469

14. Ferreira PL (2000) [Development of the Portuguese version of MOS SF-36. Part I. Cultural and linguistic adaptation]. Acta Med Port 13:55-66

15. Ferreira PL (2000) [Development of the Portuguese version of MOS SF-36. Part II -Validation tests]. Acta Med Port 13:119-127

16. Flandry F, Hunt JP, Terry GC et al (1991) Analysis of subjective knee complaints using visual analog scales. Am J Sports Med 19:112-118

17. Terwee CB, Bot SD, de Boer MR et al (2007) Quality criteria were proposed for measurement properties of health status questionnaires. J Clin Epidemiol 60:34-42

18. Nunnally J, Bernstein I (1994) Psychometric theory, 3rd ed. McGraw-Hill, New York

19. Fermanian J (1984) [Measuring agreement between 2 observers: a quantitative case]. Rev Epidemiol Sante Publique 32:408-413

20. Husted JA, Cook RJ, Farewell VT et al (2000) Methods for assessing responsiveness: a critical review and recommendations. J Clin Epidemiol 53:459-468

21. Bizzini M, Gorelick M (2007) Development of a German version of the knee outcome survey for daily activities. Arch Orthop Trauma Surg 127:781-789

22. Davies GM, Watson DJ, Bellamy N (1999) Comparison of the responsiveness and relative effect size of the western Ontario and McMaster Universities Osteoarthritis Index and the short-form Medical Outcomes Study Survey in a randomized, clinical trial of osteoarthritis patients. Arthritis Care Res 12:172-179

23. Wright JG, Young NL (1997) A comparison of different indices of responsiveness. J Clin Epidemiol 50:239-246 